\title{
Well-being of obese individuals: therapeutic perspectives
}

The history of pharmacologic treatment of obesity is characterized by too much focus on efficacy and too little on safety, which has led to withdrawals of drugs from the market after serious adverse events. The development of new drug targets for the management of obesity will definitively need to address effects of the CNS and overall cardiovascular safety in the early stages in order to avoid the mistakes from the past. For instance, weight loss can increase the symptoms of depression and promote a state of psychobiological vulnerability favoring weight regain. Body-weight management should then seek a balance between the health benefits of weight loss and its potential risks, a 'zone' that is associated with an optimal psychobiological well-being.

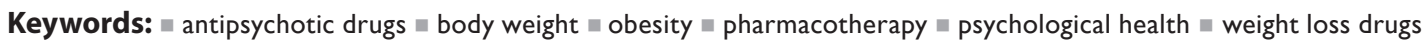

There is no doubt that the prevalence of obesity has increased worldwide in recent decades. It is also widely agreed that the problem of obesity is multifaceted and requires a combination of therapies in order to be managed. Owing to the fact that most obese individuals are insufficiently successful in their attempts to lose weight with diets, exercise and lifestyle modifications, anti-obesity drugs have a role to play in obesity management, and can enhance adherence to weight-loss programs [1]. In addition, drug therapy is easier to deliver in real-life settings, given that primary care physicians are the primary providers of obesity treatment.

In general, obese individuals can expect weight loss of $8-10 \%$ from baseline provided that they adhere to the weight-loss program and take medications regularly [2]. It is now well recognized that even modest weight loss has many health-related benefits, including an improved metabolic profile as well as better psychological well-being. However, negative psychological attributes can be associated with weight loss, particularly when individuals reach a state of resistance to fat loss (plateau).

In the field of psychiatry, weight gain is a common side effect for patients receiving antipsychotic drugs [3]. Although the mechanisms responsible for this antipsychotic-induced weight gain remain speculative, they seem to promote both an increase in food intake as well as a decrease in total energy expenditure. For the physiologist, fat gain is not considered a problem perse, but rather an unfortunate biological adaptation aimed at restoring body homeostasis [4].
Indeed, the fact that fat gain is related to a facilitation of appetite control [5] and an increase in thermogenesis [6] represents a solution promoting the long-term recovery of body-weight stability. The obvious question is then the following; For a given individual, what is the body-weight range that is compatible with psychological health? Our recent research experience has significantly contributed to determining the 'zone' associated with optimal psychobiological well-being.

\section{Weight loss up to resistance to fat loss \& well-being}

It is well recognized that an overweight state and obesity carry a social stigma that may contribute to higher rates of anxiety, depression and low self-esteem [7]. Depression may contribute to weight gain and obesity and, vice-versa. Globally, the majority of studies report the beneficial influence of weight reduction on mental well-being and health-related quality of life [8]. However, they fail to mention the possible psychological costs associated with weight loss. We believe that such negative psychological costs require a cautionary approach.

We have recently published results from a weight-loss intervention, which consisted of a supervised diet $(-700 \mathrm{kcal} /$ day $)$ and an aerobic exercise program up to a state of resistance to further fat loss. This intervention resulted in a significant increase in depression symptoms after a $10 \%$ weight loss [9], an increase that was more evident at plateau [10]. We have also been able to link the increase in depression symptoms with an increased restraint of eating, hypoglycemia at the
Jean-Philippe Chaput ${ }^{\dagger 1}$ \& Angelo Tremblay ${ }^{2}$

'Department of Human Nutrition, Faculty of Life Sciences,

University of Copenhagen Rolighedsvej 30,DK-1958, Frederiksberg C, Denmark

${ }^{2}$ Division of Kinesiology, Department of Social and Preventive Medicine,

Faculty of Medicine, Laval University, Quebec City, Quebec, Canada, GIK 7P4

${ }^{\dagger}$ Author for correspondence:

Tel.: +4535332551

Fax: +4535332483

E-mail: jepc@life.ku.dk 
end of an oral glucose challenge and a decrease in total triiodothyronine (T3) and free thyroxine (fT4) levels [9-11]. These results suggest that bringing weight loss up to a certain level has the potential to destabilize body homeostasis and induce a psychobiological vulnerability favoring weight regain.

For health professionals, these observations indicate that body-weight management should maintain a reasonable balance between the health benefits and the potential negative consequences associated with weight loss (FIGURE I).

\section{Antipsychotic drug treatment \& weight gain}

The introduction in the 1990 s of the second generation, 'atypical', antipsychotic drugs has been helpful in diminishing the problematic extrapyramidal side effects (e.g., parkinsonian symptoms) as observed with the use of older antipsychotic drugs. Nevertheless, neuroimaging studies determining drug occupancy of dopamine D2 receptors have shown that substantial antagonism of these receptors is an essential feature of effective antipsychotic action for most, if not all, of both classical and atypical antipsychotic drugs [12]. While antipsychotic drugs do improve the outlook for many patients with schizophrenia and are widely used, their efficacy remains limited. Furthermore, weight gain and metabolic disturbance encountered with these agents have emerged as a major concern for clinicians nowadays, at the same time as we face an obesity epidemic.

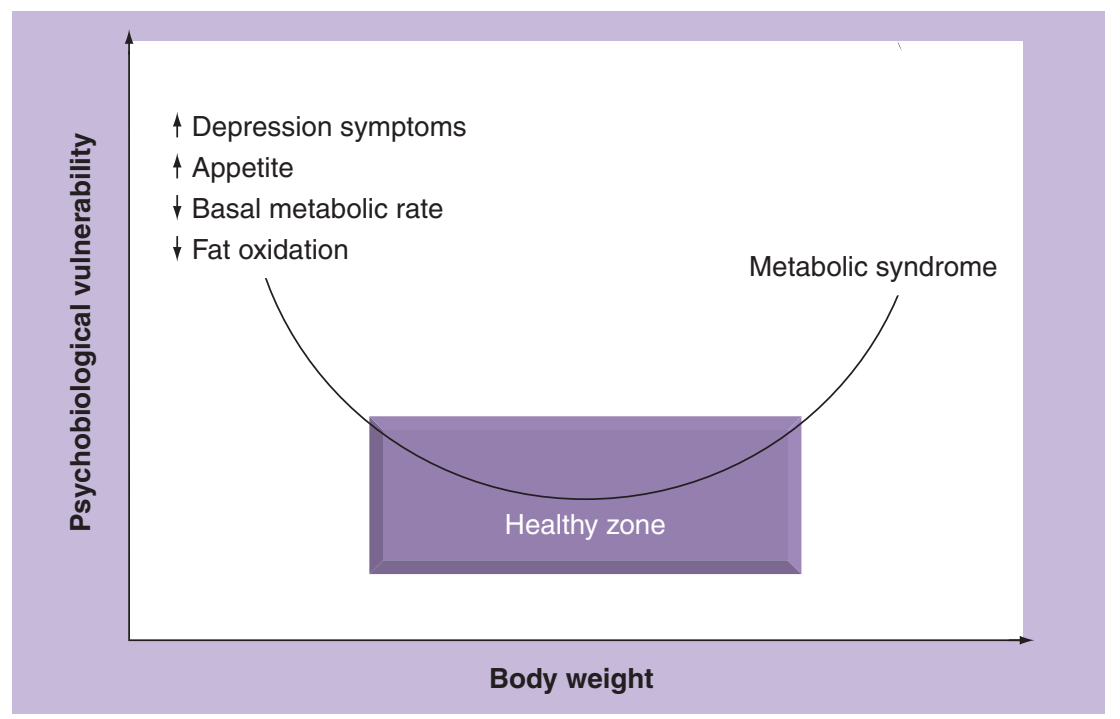

Figure 1. Notion of psychobiological well-being (healthy zone) situated between the risks of excess weight gain and excess weight loss.
While treating obesity in the general population has proven to be challenging, the challenge may be even greater for those who have gained weight in relation to a mental disorder and its pharmacological treatment. It might be indeed extremely difficult for some chronically and severely mentally ill patients who require antipsychotic treatment to eat less and exercise more when their treatment increases appetite and produces fatigue and sedation, and their illness decreases motivation and limits social interactions and activities. Additionally, their typically strained financial resources and social circumstances often limit their access to lowcalorie foods and preclude membership in health clubs, access to exercise equipment and dietary counseling or education. Such general healthpromoting activities are rarely provided in contemporary psychiatric clinical services or covered by health insurance programs for psychiatric illness, despite support for their integration into mental health services [13].

Recently, we have shown that body weight and metabolic-risk profile in patients receiving atypical antipsychotics can be managed with a behavioral weight-control program including physical activity [14]. Indeed we highlighted the importance of physical exercise designed specifically for patients on antipsychotic treatment and found that such a program is feasible and effective and the results are highly encouraging with a high rate of adherence. These results agree with those from meta-analyses showing that the effects of physical exercise are similar to those of psychotherapeutic interventions in the management of depressive symptoms $[15,16]$. In addition, exercise can alleviate secondary symptoms such as low self-esteem and social withdrawal [17]. Research also suggests that exercise is well accepted by people with serious mental illness and is often considered one of the most valued components of treatment [18]. Because individuals with serious mental illness are significantly less active than the general population, physical activity as an adjunct treatment for individuals with serious mental illness is highly desirable.

Beyond the need for a better management of antipsychotic-induced weight gain in psychiatric patients, particularly by targeting individuals prior to weight gain, one cannot exclude the possibility that an increase in body fat might be a necessary adaptation to recover psychobiological stability when one is exposed to a medication that stimulates appetite and produces sedation. Given the therapeutic value of 'comfort food' 
(i.e., palatable or energy-dense food) on wellbeing, we propose that restrictive diets may even be counter-productive for these patients. After all, George Bernard Shaw said, when talking about the pleasure of eating, that "there is no love sincerer than the love of food" (Man and Superman, 1903).

\section{Future of medicinal chemistry for obesity: a global approach}

The development of drug treatments for obesity has focused too much on efficacy and too little on safety, which has led to withdrawals of drugs from the market after serious adverse events have arisen (TABLE I) [19]. A recent example is rimonabant, a cannabinoid-receptor antagonist, which was withdrawn from the market in Europe after the discovery of an increased risk of depression, anxiety and suicidal ideation [20]. These serious adverse effects were class effects and the development of other compounds of the same class that were in Phase III studies had to be terminated. In January 2010, the weight-loss drug sibutramine (serotonin and norepinephrine reuptake inhibitor) has been withdrawn from the European market after concerns about a raised risk of heart attacks and strokes [101]. This experience highlights the need for new drugs to use novel mechanisms of action in order to avoid the serious side effects seen in the past. Furthermore, it will be important to pay attention to the CNS effects and the overall safety profile of these potential new compounds in the early stages of clinical development.

Despite considerable gains in knowledge over the past years, scientists have not enjoyed much success in translating such knowledge into finding better drug therapies. Numerous drug candidates with novel mechanisms of action have either not achieved clinically meaningful efficacy or failed to meet the safety expectation of regulatory authorities. Possible reasons for the failure of anti-obesity drugs (e.g., compensatory mechanisms that overcome pharmaceutical manipulations) are explained in detail elsewhere [21]. Importantly, the failure of new drug candidates emphasizes that the problem of obesity is very complex and that there are numerous causes of excess weight. A drug specifically targeting a particular pathway might not achieve a robust therapeutic effect in an obese individual, if this specific person's obesity is primarily related to the actions of another pathway, on which this specific drug does not have a major impact. Hence, the large interindividual variation in response to
Table 1. History of drug treatments for obesity by date of approval/withdrawal.

\begin{tabular}{|lll|}
\hline Date & Drug & Serious adverse events \\
\hline $1893 / 1949$ & Thyroid hormone & Hyperthyroidism \\
\hline $1933 / 1935$ & Dinitrophenol & Cataracts and neuropathy \\
\hline $1937 / 1971$ & Amfetamine & Addiction and psychosis \\
\hline $1965 / 1972$ & Aminorex & Pulmonary hypertension \\
\hline $1973 / 1997$ & Fenfluramine plus phentermine & Cardiac valvular insufficiency \\
$1960 / 2000(\text { USA })^{\dagger}$ & Phenylpropanolamine & Hemorrhagic stroke \\
\hline $2006 / 2009$ & Rimonabant & $\begin{array}{l}\text { Depression and } \\
\text { suicidal ideation }\end{array}$ \\
\hline $1997 / 2010$ & Sibutramine & Cardiovascular disease \\
\hline $\begin{array}{l}{ }^{+} \text {Phenylpropanolamine is still available in some European countries. } \\
\text { Reproduced with permission from [19]. }\end{array}$
\end{tabular}

drug-induced weight loss seems to be attributed to the fact that we do not target the cause(s) of the problem.

In the future, health professionals should be able to count on early diagnosis of individuals at risk of developing long-term metabolic problems and obesity, or for not responding adequately to clinical interventions. We believe that such personalized therapy has the potential to improve the chances of success and decrease the number of irrelevant pharmacologic interventions.

Orlistat, an inhibitor of pancreatic and gastrointestinal lipases, is the only drug currently approved for long-term treatment of obesity in Europe. Side effects due to the mode of action include oily spotting, liquid stools, fecal urgency or incontinence, flatulence and abdominal cramping. In addition, lipid-soluble vitamins (A, D, E and K) should be replaced. Pooled results of 11 prospective randomized controlled trials show that patients treated with Orlistat displayed a $2.7-\mathrm{kg}$ or $2.9 \%$ greater reduction in weight than placebo-treated patients after a 1-year follow-up [22]. In addition to the modest efficacy of Orlistat, less than $10 \%$ of patients still take the drug after 1 year and only $2 \%$ of them after 2 years [23]. Whereas factors such as tolerability and affordability might also be reasons, failure of Orlistat in real-life settings is most likely attributable to the fact that obese patients expect to lose an average of approximately $25 \%$ of their body weight after 1 year of treatment [24] and the efficacy of Orlistat falls far below the expectations of the patients.

A total of four sympathomimetic medications are also approved for short-term use (<12 weeks) for weight loss. Phentermine, diethylpropion, phendimetrazine and benzphetamine are all amphetamine-derived appetite suppressants that centrally release dopamine and noradrenaline [25]. 
These drugs stimulate the CNS and increase blood pressure, pulse rate and release of glycerol and free fatty acids. Insomnia, dry mouth and constipation are the main side effects of these drugs. Phentermine and diethylpropion are more commonly prescribed due to their reduced potential for addiction [25]. However, the short-term use of these sympathomimetic drugs may limit a long-term treatment of obesity and, here again, their efficacy and adverse effects do not please all patients.

At present, there are three new weight-loss drugs on the horizon. Qnexa ${ }^{\circledR}$, developed by Vivus, has proven to be the most effective, with a 1-year placebo-subtracted weight loss of 6.6-8.6\% [102]. Additionally, weight loss relative to the baseline of $10.5-13.2 \%$ was seen for patients completing the trial. Qnexa is a mixture of two existing drugs, an anticonvulsant (topiramate) and an amphetamine (phentermine). Unfortunately, there are side effects with this drug (memory and concentration problems for instance), which caused many participants to drop out of the study. The second weight-loss drug, Contrave (Orexigen ${ }^{\circledR}$ Therapeutics), is a mixture of two well-known compounds, naltrexone and bupropion. Research has shown weight loss of $5.0-6.1 \%$, with side effects such as nausea [26]. The third candidate, Lorcaserin from Arena Pharmaceuticals, acts selectively on the central serotonin $2 \mathrm{C}$ receptors. Lorcaserin has less side effects than the previous two, but weight loss has been reported to be modest (weight-loss efficacy slightly less than or equivalent to that of orlistat) [27]. Given the history, we will need to be completey sure about the safety of these new weight-loss drugs, because these medications must be taken for a number of years.

What is then the future of medicinal chemistry for obesity? Despite new molecular targets and several novel agents under active

\begin{tabular}{|c|c|c|}
\hline $\begin{array}{l}\text { Product name/ } \\
\text { company }\end{array}$ & Product type & Code \\
\hline Qnexa $^{\circledR}$ & Vivus & Phentermine and topiramate \\
\hline Contrave & Orexigen ${ }^{\circledR}$ Therapeutics & Bupropion and naltrexone \\
\hline Lorcaserin & Arena Pharmaceuticals & $5-\mathrm{HT}_{2 \mathrm{C}}$ agonist \\
\hline Tesofensine & NeuroSearch & NA/DA/5HT reuptake inhibitor \\
\hline Liraglutide & Novo Nordisk & GLP-1 analogue \\
\hline Velneperit & Shionogi & NPY Y5 receptor antagonist \\
\hline Empatic & Orexigen ${ }^{\circledR}$ Therapeutics & Bupropion and zonisamide \\
\hline Dov 21947 & Dov Pharmaceuticals & NA/DA/5-HT reuptake inhibitor \\
\hline
\end{tabular}

development (TABLE 2), optimal treatment of obesity will likely require combinations of therapies. This obviously implies the adherence to a healthy active living in order to optimize the efficacy of the drug treatment as well as to improve overall well-being. It is of interest to mention that the side effects associated with the use of Orlistat are attenuated if the patients follow a low-lipid diet in concomitance with the drug treatment. Additionally, the prevention of undesirable effects of this medication by a low-fat diet not only improves the well-being of patients, but is also likely to accentuate weight loss. Likewise, the cardio-stimulatory effects of the weight loss drug Sibutramine ${ }^{\circledR}$ have been shown to be offset by a physical exercise program [28].

\section{Conclusion}

Pharmaceutical approaches to the management of obesity tend to focus on simple end points, such as the absolute reduction in body weight and normalization of biomarkers of obesity-related disease. This approach directs the attention at the consequences rather than the causes of obesity and fails to exploit knowledge of the psychological factors that influence appetite. Obesity is typically a consequence of overconsumption driven by an individual's natural sensitivity to food stimuli and the pleasure derived from eating. As recently proposed, these processes should be effective targets for pharmacotherapy and behavioral analysis can identify drugs that selectively affect the desire to eat, enjoyment of eating, satiation or postmeal satiety [29].

Body-weight management should be performed with the preoccupation of maintaining a reasonable balance between the health benefits of weight loss and its potential risks. Weight-loss drugs are appropriate only as an addition - not a replacement for - healthy active living. As illustrated in Figure I, health professionals should try to keep their patients in the 'zone' associated with an optimal psychobiological well-being.

\section{Financial \& competing interests disclosure}

The authors have no relevant affiliations or financial involvement with any organization or entity with a financial interest in or financial conflict with the subject matter or materials discussed in the manuscript. This includes employment, consultancies, honoraria, stock ownership or options, expert testimony, grants or patents received or pending, or royalties.

No writing assistance was utilized in the production of this manuscript. 


\section{Bibliography}

1 Kaplan LM. Pharmacologic therapies for obesity. Gastroenterol. Clin. North Am. 39, 69-79 (2010)

2 Bray GA. Medications for obesity: mechanisms and applications. Clin. Chest. Med. 30, 525-538 (2009).

3 Muench J, Hamer AM. Adverse effects of antipsychotic medications. Am. Fam. Physician 81, 617-622 (2010).

4 Tremblay A, Doucet E. Obesity: a disease or a biological adaptation? Obes. Rev. 1, 27-35 (2000).

5 Gilbert JA, Drapeau V, Astrup A, Tremblay A. Relationship between dietinduced changes in body fat and appetite sensations in women. Appetite 52, 809-812 (2009).

6 Doucet E, St-Pierre S, Alméras N, Després JP, Bouchard C, Tremblay A. Evidence for the existence of adaptive thermogenesis during weight loss. Br. J. Nutr. 85, 715-723 (2001).

7 Puhl R, Brownell KD. Bias, discrimination, and obesity. Obes. Res. 9, 788-805 (2001).

8 Fontaine KR. Weight loss and health-related quality of life. Am. J. Manag. Care 7 , 926-927 (2001).

9 Chaput JP, Drapeau V, Hetherington M, Lemieux S, Provencher V, Tremblay A. Psychobiological impact of a progressive weight loss program in obese men. Physiol. Behav. 86, 224-232 (2005).

10 Chaput JP, Drapeau V, Hetherington M, Lemieux S, Provencher V, Tremblay A. Psychobiological effects observed in obese men experiencing body weight loss plateau. Depress. Anxiety 24, 518-521 (2007).

11 Chaput JP, Arguin H, Gagnon C, Tremblay A. Increase in depression symptoms with weight loss: association with glucose homeostasis and thyroid function. Appl. Physiol. Nutr. Metab. 33, 86-92 (2008).

12 Reynolds GP, Kirk SL. Metabolic side effects of antipsychotic drug treatment pharmacological mechanisms. Pharmacol. Ther. 125, 169-179 (2010).

13 Richardson CR, Faulkner G, McDevitt J, Skrinar GS, Hutchinson DS, Piette JD. Integrating physical activity into mental health services for persons with serious mental illness. Psychiatr. Serv. 56, 324-331 (2005).

14 Poulin MJ, Chaput JP, Simard V et al. Management of antipsychotic-induced weight gain: prospective naturalistic study of the effectiveness of a supervised exercise programme. Aust. NZ J. Psychiatry 41, 980-989 (2007).

15 Lawlor DA, Hopker SW. The effectiveness of exercise as an intervention in the management of depression: systematic review and meta-regression analysis of randomised controlled trials. BMJ 322, 763-767 (2001).

16 Craft LL, Landers DM. The effect of exercise on clinical depression and depression resulting from mental illness: a mata-analysis. J. Sports Exerc. Psychol. 20, 339-357 (1998).

17 Faulkner G, Biddle S. Exercise as an adjunct treatment for schizophrenia: a review of the literature. J. Ment. Health 8, 441-457 (1999).

18 Martinsen E. The effects of exercise on mental health in clinical populations. In: European Perspectives on Exercise and Sport Psychology. Biddle S (Ed.) Human Kinetics, Stanningley, UK, 185-211 (1995).

19 Astrup A. Is cardiometabolic risk improved by weight-loss drugs? Lancet 376(9741), 567-568 (2010).

20 Christensen R, Kristensen PK, Bartels EM, Bliddal H, Astrup A. Efficacy and safety of the weight-loss drug rimonabant: a meta-analysis of randomised trials. Lancet 370, 1706-1713 (2007).

21 Gadde KM, Allison DB. Combination therapy for obesity and metabolic disease. Curr. Opin. Endocrinol. Diabetes Obes. 16, 353-358 (2009).

22 Padwal R, Li SK, Lau DC. Long-term pharmacotherapy for overweight and obesity: a systematic review and meta-analysis of randomized controlled trials. Int. J. Obes. Relat. Metab. Disord. 27, 1437-1446 (2003).

23 Padwal R, Kezouh A, Levine M, Etminan M. Long-term persistence with orlistat and sibutramine in a population-based cohort. Int. J. Obes. 31, 1567-1570 (2007).
24 Foster GD, Wadden TA, Vogt RA, Brewer G. What is a reasonable weight loss? Patients' expectations and evaluations of obesity treatment outcomes. J. Consult. Clin. Psychol. 65, 79-85 (1997).

25 Robinson JR, Niswender KD. What are the risks and the benefits of current and emerging weight-loss medications? Curr. Diab. Rep. 9, 368-375 (2009).

26 Greenway FL, Fujioka K, Plodkowski RA et al. Effect of naltrexone plus bupropion on weight loss in overweight and obese adults (COR-I): a multicentre, randomized, double-blind, placebo-controlled, Phase 3 trial. Lancet 376(9741), 595-605 (2010).

27 Smith SR, Weissman NJ, Anderson CM et al. Multicenter, placebo-controlled trial of lorcaserin for weight management. N. Engl. J. Med. 363, 245-256 (2010).

28 Bérubé-Parent S, Prud'homme D, St-Pierre S, Doucet E, Tremblay A. Obesity treatment with a progressive clinical tri-therapy combining sibutramine and a supervised diet-exercise intervention. Int. J. Obes. 25, 1144-1153 (2001).

29 Halford JC, Boyland EJ, Blundell JE, Kirkham TC, Harrold JA. Pharmacological management of appetite expression in obesity. Nat. Rev. Endocrinol. 6, 255-269 (2010).

\section{- Websites}

101 European Medicines Agency. Questions and answers on the suspension of medicines containing sibutramine. 21 January 2010. www.ema.europa.eu/pdfs/human/referral/ sibutramine/Sibutramine_Q\&A_80917909 en.pdf

(Accessed 13 August 2010).

102 Vivus company information. www.vivus.com (Accessed 13 August 2010). 